\section{Ethics and infectious disease}

Editors: Michael J Selgelid, Margaret P Battin

\& Charles B Smith

Publisher: Wiley-Blackwell, Oxford, 2006

ISBN: 978-1-405-14596-1; paperback;

416 pages; price £20.99

In an oft-quoted statement, the Surgeon General of the United States of America, William Stewart, said in 1967: "The time has come to close the book on infectious diseases. We have basically wiped out infection in the United States." This influential comment has, however, been contradicted by subsequent events. For example, Jones et al. recently reported that from 1940 to 2004 several hundred new infectious diseases emerged, many of which are zoonotic and bacterial. ${ }^{1}$ More than half of these new diseases have emerged since the 1970s, with the 1980s being the decade with the most new infections. Clearly, infectious diseases are still an open book.

Humans and pathogens share a long co-evolution, and infections have exerted a remarkable influence on the course of historical events and human societies. This has been abundantly well documented by historians. Yet somehow, infectious diseases have not attracted a great deal of attention from bioethicists.

This volume of essays is a welcome attempt to remedy that deficit. It stems from a thematic issue of the journal Bioethics devoted to ethics and infectious disease, published in 2005. A point of departure for the book is the so-called 10:90 divide, whereby less than $10 \%$ of medical research is devoted to diseases that account for more than $90 \%$ of the global burden of disease. Driving this agenda is a concern for the most disadvantaged populations, which are disproportionately affected by infectious diseases.

Selgelid, in the introductory essay, points out that a review of the bioethics literature demonstrates a paucity of serious ethical reflection on infectious diseases. He elucidates several reasons for this neglect. First, he argues that bioethicists have been overly concerned with the ethical issues associated with the use of high technology such as reproductive technologies and intensive care units. Second, he points out that optimism has given rise to the belief that infectious diseases are no longer the dominant threat to global health, a sentiment captured by the quotation in the first paragraph of this review. Third, infectious diseases entail "otherness". Individuals with infectious diseases such as HIV/AIDS or tuberculosis are often stigmatized. The fourth, and perhaps the most important, point that he makes is the complex nature of infectious diseases. A true understanding of the implication of the impact of an infectious organism on individuals and communities requires the integration of microbiology, immunology, clinical medicine, epidemiology, psychology, geography, anthropology, zoology and many other disciplines. Unfortunately, there are few individuals who can achieve this with sufficient expertise.

The introductory essay superbly establishes the framework for those that follow. The book is extremely well laid out, the 22 chapters are pertinent, and the authors are first rate. It is divided into six parts. Part I explores the relationship between bioethics and infectious disease in three chapters that examine some of the special features of infectious diseases and the unique ethical issues pertaining to them. Part II covers ethics and infectious disease control, introducing the ethical challenges of drug-resistant tuberculosis, wildlife diseases, pandemic preparedness, and public health and the use of restrictive measures for disease control. Part III deals with the issues and dilemmas associated with the treatment of individuals with infectious disease. Part IV is a welcome contribution to the emerging and surprisingly limited literature on the ethical issues raised by mass vaccination programmes. Part $V$ focuses attention on developing countries and global health, with stimulating essays by Paul Farmer, Nicole Gastineau-Campos, Thomas Pogge and Tim Anderson.
Finally, part VI tackles the bioethical dimensions of security and bioterrorism.

Altogether this set of essays would make compelling reading for any public-health practitioner or clinician interested in infectious diseases. I can give it an unreserved recommendation. However, there is one small caveat. As timely as this book is, it already shows signs of needing updates. The emergence of extensively drug-resistant tuberculosis (XDR-TB) makes many of the book's essays on tuberculosis control no longer completely relevant, since XDR-TB raises a whole host of ethical questions that are of the utmost importance for global health. Finally, there is little coverage of hospital-borne infections, such as methicillin-resistant Staphylococcus aureus (MRSA) and vancomycin-resistant enterococci (VRE), which raise significant ethical issues. The concept linking these last two topics is drug resistance, a largely human-made phenomenon that has had a catastrophic impact on hospital care and the health of communities.

In short, infectious diseases are still with us and they are likely to remain an eternal aspect of human existence. It is time that the bioethical issues relevant to the impact and control of infectious diseases on individuals, communities and populations receive the type of robust ethical reflection that other aspects of health care have received. The publication of this book is a welcome addition to the bioethics literature; it will be particularly suitable for use in postgraduate courses on infectious diseases, public-health ethics or clinical ethics. The authors are to be commended for their attention to this topic and for inviting some of the best minds in bioethics to address some of the most difficult, but neglected, ethical issues in public health and medicine.

\section{Ross Upshur}

\section{Reference}

1. Jones KE, Patel N, Levy M, Storeygard A, Balk D, Gittleman J, et al. Global trends in emerging infectious diseases. Nature 2008;451:990-3.

\footnotetext{
a Joint Centre for Bioethics, University of Toronto, Toronto, ON, Canada.

Correspondence to Ross Upshur (e-mail: ross.upshur@utoronto.ca).
} 